\title{
Atypical Presentation of Glioblastoma Multiforme
}

\author{
Wissam Al-Janabi, Renee Krebs, Ximena Arcila-Londono, Iram Zaman, Bashiruddin K. Ahmad \\ Neurology Department, Henry Ford Health System, Detroit, MI, USA
}

How to cite this article: Al-Janabi W, Krebs R, Arcila-Londono X, Zaman I, Ahmad BK. Atypical presentation of glioblastoma multiforme. EJCRIM 2018;5: doi:10.12890/2018_000954.

Conflicts of Interests: The Authors declare that there are no competing interests.

This article is licensed under a Commons Attribution Non-Commercial 4.0 License

\section{ABSTRACT}

Background: Glioblastoma multiforme (GBM) is a highly malignant glial tumour classified by the World Health Organization (WHO) as a stage IV astrocytoma. It varies in shape and size and can be cystic, vascular and necrotic. It often appears as a ring-enhancing lesion on magnetic resonance imaging (MRI). The most common symptoms of GBM, such as headache, vomiting and seizures, are due to increased intracranial pressure. The objective of this case report is to describe an atypical presentation of GBM.

Case Report: A 53-year-old woman of Italian origin presented with a 2-week history of lack of coordination in her hands and some difficulty in speech. Electromyography for assessment of her arms and cranial bulbar function was normal. However, 2 days later, the patient presented to the emergency department with progressive weakness in her left arm and leg as well as difficulty in speech. Mild left facial asymmetry was noted. A brain MRI revealed a right frontal mass. Stereotactic surgical resection was performed 2 days later, and biopsy confirmed the diagnosis of GBM. Although headache and other features of raised intracranial pressure are the most common initial symptoms of GBM, any atypical neurological or psychiatric presentation in an adult patient should raise suspicion for this tumour.

Conclusion: Careful analysis of an adult with atypical signs and symptoms along with thorough review of radiological tests will facilitate early diagnosis of dangerous tumours such as GBM.

\section{LEARNING POINT}

- An adult patient with symptoms that do not conform to a neurological condition should be investigated for a brain tumour.

- Careful history taking and examination are essential for reaching the correct diagnosis as soon as possible.

- Meticulous review of radiological images in order to detect subtle changes in brain anatomy is essential.

\section{KEYWORDS}

Glioblastoma multiforme, primary care physician, World Health Organization, emergency department, cerebrospinal fluid

\section{BACKGROUND}

Glioblastoma multiforme (GBM) is a primary brain tumour which is characterized by a rapidly growing mass that varies in size and shape, and accounts for $70 \%$ of all primary brain tumours. It is classified by the WHO as a highly malignant tumour ${ }^{[1-3]}$. Although GBM may present as multiple lesions, it is usually a just single mass ${ }^{[2]}$. Headache, vomiting and seizure are the most common presentations ${ }^{[2,4,5]}$. The GBM can double in size in 2 days to several weeks, which gives it a dire prognosis, with a median survival time of 9.9 months if treated with surgery and radiotherapy with or without chemotherapy. It is twice as common in white as in black patients ${ }^{[2,5-7]}$ and is also slightly more frequent in males than females. It is often located in the supratentorial region of the brain with extensive necrosis ${ }^{[5,6]}$. 
About $5 \%$ of patients with GBM have a family history, and some cases are linked to rare syndromes, such as Li-Fraumeni syndrome, neurofibromatosis I and II, or Turcot's syndrome ${ }^{[6]}$. If the GBM tumour involves the corpus callosum, then it is called 'butterfly glioma', and usually metastasizes to the occipital and temporal lobes bilaterally ${ }^{[2,6]}$. Good prognostic features for GBM are younger age, lateral tumour localization, complete macroscopic resection, and abundant giant cells (gigantocellular glioblastoma) ${ }^{[7,8]}$. The brain MRI may not show a typical mass. Indeed, deceiving features of a demyelinating process may be seen. Hence GBM should be considered in a middle-aged patient with an atypical presentation who does not respond to the usual treatment for the suspected condition ${ }^{[9]}$. GBM can also occur in

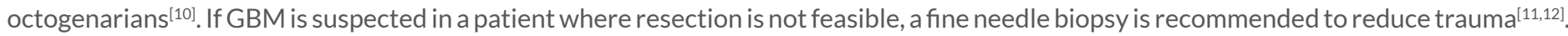
GBM can spread directly to adjacent parts of the brain or extracranially via the CSF ${ }^{[13]}$. Both GBM and its treatment can cause neurological, cognitive or psychiatric symptoms ${ }^{[14]}$.

\section{CASE REPORT}

A 53-year-old woman presented to her primary care physician complaining of feeling anxious, with panic at times. She was having difficulty finding words and had bilateral hand and wrist pain, which had largely improved although there was still some lack of coordination. She also had trouble getting out of the bed and getting dressed. However, she denied headache, nausea or vomiting. The patient described her anxiety symptoms as insomnia, shortness of breath and feeling 'closed in'. She had experienced these symptoms for a few years but they had got worse over the past month. She denied suicidal and homicidal ideation. The patient felt more comfortable sleeping during the day, but this caused her to stay up late at night. She had a past medical history of allergic rhinitis, asthma, hyperglycaemia and hyperlipidaemia. She had a positive family history of anxiety. Physical examination showed normal vital signs, mild nasal congestion, quiet and clear speech, possible mild bilateral thenar atrophy, a mildly positive Phalen's test, and a negative Tinel sign. Mild left facial asymmetry was also noted. Electromyography was normal.

Two days later, the patient presented to the emergency department complaining of progressive left-sided weakness and difficulty in speech. A CT scan of the head to rule out stroke surprisingly showed a mass in the right frontal lobe. MRI confirmed the presence of this mass which extended to the corpus callosum. The mass measured $5.7 \mathrm{~cm}$ by $3.8 \mathrm{~cm}$ (Fig. 1). CT scans of the chest, abdomen and pelvis were negative for any metastases.
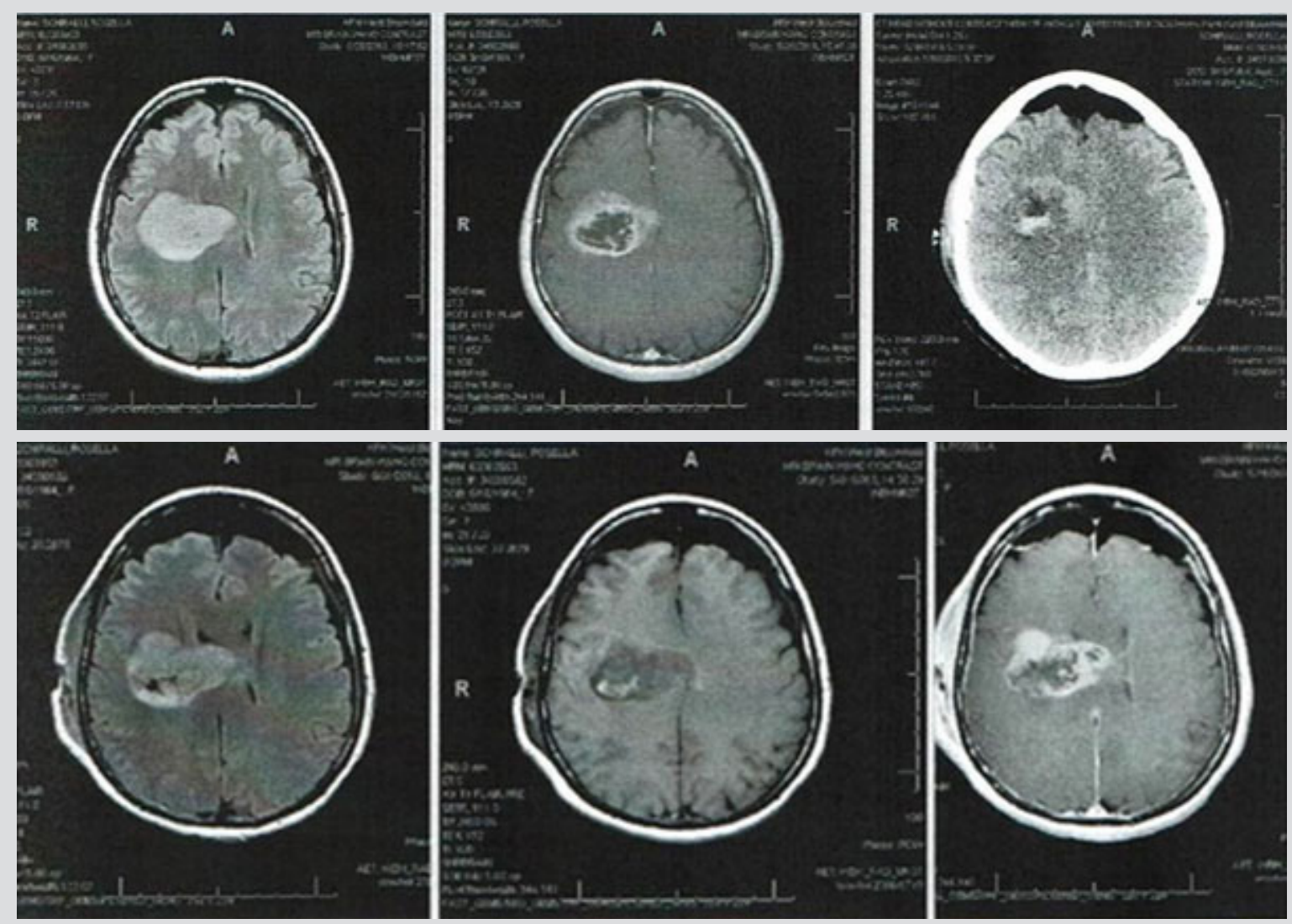

Figure 1. Magnetic resonance imaging clearly shows the right frontal lobe mass extending to the corpus callosum 
A right frontal stereotactic craniotomy revealed a multicystic, vascular, necrotic mass which was soft in many areas. Frozen sections and biopsy slides (Fig. 2) showed an infiltrating astrocytic tumour with necrosis, microvascular proliferation, and brisk mitotic activity. The tumour cells were strongly immunoreactive to GFAP, and negative for Cam 5.2 and IDH1 R132H mutant antibody. Neurofilament immunostaining highlighted the infiltrating nature of this tumour. P53 immunostaining showed nuclear expression in a minor subset of the tumour cells. All immunostaining was performed with appropriate controls on separate slides. The final diagnosis was GBM of the brain.
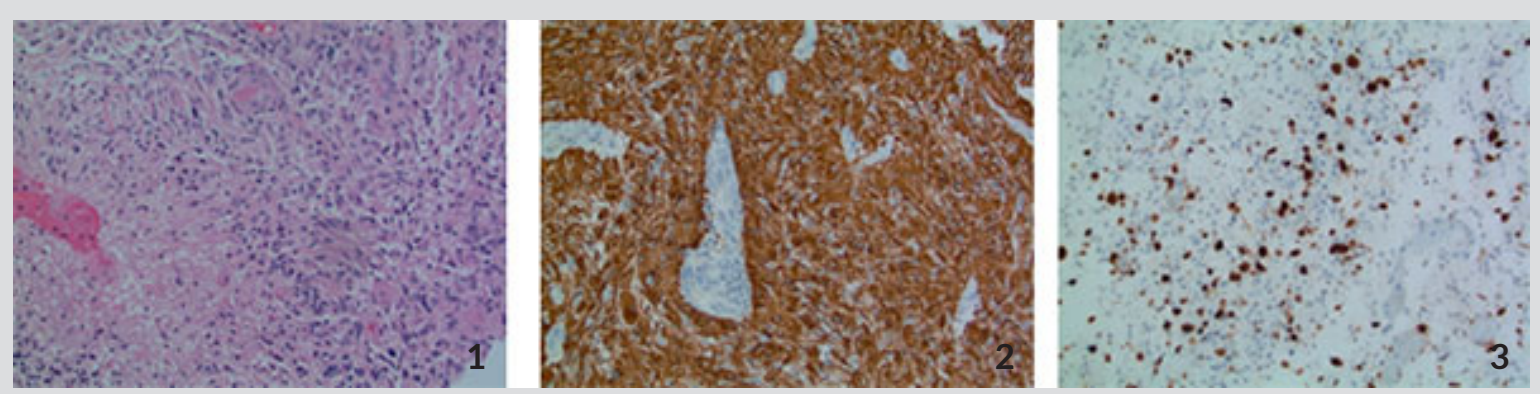

Figure 2. Histopathology: (1) H\&E stain reveals the tumour with necrosis; (2) GFAP immunostaining is positive for tumour cells; a blood vessel with negative staining shows microvascular proliferation; (3) MIB-1 immunostaining of numerous tumour cells. Molecular information: MIB proliferation index $20 \%$. IDH1 R123H (IHC): negative. IDH1 (NGS): negative; EGFRvIll mutation: negative; MGMT promoter methylation: negative

\section{DISCUSSION}

The first case of GBM was reported in 1880 by Bradley ${ }^{[13]}$. GBM is one of the deadliest adult tumours and has a grave prognosis ${ }^{[1,2]}$. Although it usually presents as a supratentorial tumour, rare cases involving the brainstem and cerebellum have been reported ${ }^{[9,10]}$. Owing to its shape on MRI, which is often described as a ring-enhancing lesion, GBM is sometimes misdiagnosed as an infection or inflammatory process ${ }^{[1,9]}$. GBM is highly metastatic via CSF or spread to adjacent regions ${ }^{[1,13]}$. In the present case, only a single large right frontal mass was identified, with all other screening for satellite lesions being negative. The corpus callosum was involved in this case, but no hydrocephalus or raised intracranial pressure was detected. The swift progression of the patient's symptoms might have been due to rapid growth of the GBM mass. Previous studies have shown that it can take from only 2 days to several weeks for the tumour to double in size ${ }^{[7]}$. Patients survival time is also related to tumour doubling time ${ }^{[7,8]}$. Patients with glioma can exhibit psychiatric symptoms, such as personality and behavioural changes, hallucinations, mood issues or even psychosis ${ }^{[14]}$. In the present case, the patient's initial complaints of anxiety, left-hand lack of coordination (which developed into left-hand weakness) and difficulty in speech were unlike the usual symptoms of brain tumour patients (early morning headache, vomiting or vision disturbance) and more like those of motor neuron disease (MND). However, the patient's EMG result ruled out MND and a CT scan 2 days later revealed the GBM mass in the right frontal lobe. The patient is still alive until this moment.

\section{CONCLUSION}

A careful history taking and physical examination of patients with atypical neurological, cognitive or psychiatric symptoms that do not conform with known neurological or psychiatric disorders along with meticulous review of any radiological tests will help early detection of serious tumours. Neurologists should always keep GBM in mind, especially when a patient presents with unusual symptoms that do not suggest well-known neurological conditions. 


\section{REFERENCES}

1. Daroff R, Jankovic J, Mazziotta J, Pomeroy S. Bradley's neurology in clinical practice. 7th ed. London: Elsevier Health Sciences; 2015.

2. Zhang YY, Ruan LX, Zhang S. Rapid progression of glioblastoma multiforme: a case report. Oncol Lett 2016;12:4803-4806.

3. Louis DN, Ohgaki H, Wiestler OD, Cavenee WK, editors. WHO classification of tumours of the central nervous system. Vol 1. 4th ed. Lyon: IARC Press; 2007.

4. Sanli AM, Turkoglu E, Dolgun H, Sekerci Z. Unusual manifestations of primary glioblastoma multiforme: a report of three cases. Surg Neurol Int 1;87:2010.

5. de Castro-Costa CM, de Araújo RW, de Arruda MA, de Araújo PM, de Figueiredo EG. Increased intracranial pressure in a case of spinal cervical glioblastoma multiforme. Analysis of these two rare conditions. Arq Neuropsiquiatr 1994;52:64-68.

6. Wen PY, Kesari S. Malignant gliomas in adults. N Engl J Med 2008;359:492-507.

7. Furneaux CE, Marshall ES, Yeoh K, Monteith SJ, Mews PJ, Sansur CA, et al. Cell cycle times of short-term cultures of brain cancers as predictors of survival. Br J Cancer 2008;99:1678-1683.

8. Cvetkoivč-Dožič D, Skender-Gazibara M, Dožič S. Morphological and molecular features of diffuse infiltrating astrocytoma. Arch Oncol 2004;12:38-39.

9. Lakhan SE, Harle L. Difficult diagnosis of brainstem glioblastoma multiforme in a woman: a case report and review of the literature. J Med Case Rep $2009 ; 3: 87$.

10. Levine SA, McKeever PE, Greenberg HS. Primary cerebellar glioblastoma multiforme. J Neurooncol 1987:5:231-236.

11. Kleihues P, Burger PC, Collins VP, Cavenee WK, editors. World Health Organization classification of tumours. Pathology and genetics of tumours of the nervous system. Lyon: IARC Press; 2000.

12. Katsetos CD, Dráberová E, Legido A, Dráber P. Tubulin targets in the pathobiology and therapy of glioblastoma multiforme. II. gamma-Tubulin. J Cell Physiol 2009;221:514-520.

13. Bradley WL. Case of gliosarcomatous tumors of the brain. Proc Conn Med Soc 1880;2:39-41.

14. Boele FB, Rooney AR, Grant, RG, Klein MK. Psychiatric symptoms in glioma patients: from diagnosis to management. Neuropsychiatr Dis Treat 2015;11:1413-1420. 